\title{
Paulo Freire y su legado político pedagógico en la sistematización de experiencias emancipatorias
}

\author{
* María Mercedes Ruiz Muñoz \\ Universidad Iberoamericana (México). \\ mercedes.ruiz@ibero.mx
}

Fecha de recepción: 6 de abril de 2020. Fecha de aceptación: 30 de mayo de 2020.

Nadie educa a nadie -nadie se educa a sí mismo-, los hombres se educan entre sí con la mediación del mundo.

Paulo Freire (2019: 61).

\begin{abstract}
Resumen
En este trabajo se presenta la recuperación del pensamiento freireano a la luz de la reconstrucción de experiencias a partir de la voz de los sujetos. La marca de Paulo Freire en el campo de la investigación educativa juega de manera significativa en la producción del conocimiento y en los saberes que emergen de los procesos de constitución de sujetos. La mirada analítica de la imbricación de lo político y lo pedagógico opera como una lógica de intelección para analizar los procesos educativos que se dan más allá de la escuela y al interior de la misma. De acuerdo con Paulo Freire, no se puede aludir a lo educativo sin lo político y a lo político sin lo educativo. En este sentido, la sistematización de experiencias opera bajo estos registros y otorga un sentido particular a la narrativa de los sujetos.
\end{abstract}

Palabras clave: imbricación, educación, político, pedagógico, sujetos.

\section{Paulo Freire's Pedagogical Political Legacy in the Systematization of Emancipatory Experiences}

\begin{abstract}
This article presents a return to Freirean thought in light of the reconstruction of experiences based on individual accounts. Paulo Freire's influence in the field of educational research plays a significant role in the production of knowledge and in the knowledge that emerges from individual processes of narration. The analytical view
\end{abstract}


of the interweaving of politics and pedagogy serves as an understanding rationale to study the educational processes that take place beyond and within schools. According to Paulo Freire, one cannot refer to education without considering politics, nor to politics without considering education. In this sense, the systematization of experiences operates within these spheres and attaches a unique meaning to individual narratives.

Keywords: nesting, education, political, pedagogical, subjects.

\section{A manera de introducción}

Entre las enseñanzas de Paulo Freire, la imbricación de lo político y lo pedagógico forma parte constitutiva de los procesos educativos que se dan en la institución escolar y más allá de la escuela. A partir de este posicionamiento freireano, a lo largo de nuestra trayectoria profesional, hemos constatado que los campos de la educación de jóvenes y adultos y los de los movimientos y organizaciones sociales representan ámbitos y espacios socioeducativos de investigación e intervención político-pedagógica. En este trabajo, se presenta una reflexión acerca de estos registros y sus aportes a la sistematización de experiencias educativas como una forma de investigación social, que pretende dar cuenta de las alternativas pedagógicas que emergen de los grupos organizados y de los movimientos sociales.

Por ello, nos referiremos a la educación en el sentido amplio del término, como un proceso social que ocurre en múltiples espacios socioeducativos: en las reuniones comunitarias, las asambleas estudiantiles, las protestas en la calle, la liturgia eclesial, la escuela, el salón de clases y sus pasillos, entre otros, los cuales constituyen un archipiélago educativo, y el ámbito en el que Freire innovó con su metodología de educación popular. Particularmente lo hizo con Pedagogía del oprimido (2019), cuyo método de la palabra generadora posibilitó leer el mundo y la realidad a fin de transformarlos, lo cual marcó un hito en el pensamiento pedagógico latinoamericano y otorgó sentidos políticos y pedagógicos a la educación popular y de adultos.

La obra de Paulo Freire, a cien años de su nacimiento, adquiere un significado más que relevante no solo por su aporte a la educación popular y de adultos, sino por las marcas que dieron sentido al pensamiento de la pedagogía crítica que se fue configurando en América Latina a partir de la década de 1960 y que, hoy día, hace que la lectura del mundo y de la realidad para su transformación sea vista como una herencia de su pensamiento, en particular para las pedagogías emancipatorias actuales, que posibilitan la acción colectiva en torno al cuidado de la tierra y del medio ambiente, el respeto a los derechos humanos, a la situación de género, la etnicidad y el racismo, entre otros. En México, por ejemplo, las luchas y acciones colectivas se articulan en torno a la violencia de género, a los movimientos de Ayotzinapa por los cuarenta y tres estudiantes normalistas desaparecidos, a Más de 131 y\#Yo soy 132, entre otros.

En este escrito se analizan marcas freireanas a la luz de su obra y su resignificación, inscritas a lo largo de nuestro trabajo doctoral: la imbricación de lo político y lo pedagógico: "la educación de adultos de los sectores populares se caracteriza como una práctica educativa con sentido y pertinencia política: la lectura de la realidad, la problematización, la concientización y la transformación del mundo representan un horizonte de pensamiento que se construyó en el marco del pensamiento pedagógico latinoamericano" (Ruiz, 2005). Además, se problematiza acerca de la sistematización de experiencias en el marco de la educación popular, perspectiva investigativa cuya fuente de inspiración fue el diálogo, la concientización y la acción a partir de la voz de los propios sujetos. 


\section{Imbricación de lo político y lo pedagógico}

La noción de imbricación de lo político y lo pedagógico surge en el marco de la tesis doctoral (Ruiz, 2005), para dar cuenta de la teoría central del planteo de Paulo Freire en torno a que la educación tiene cualidades inherentes a la política, de la misma forma que la política tiene atributos educativos. En su momento, la búsqueda inicial estuvo permeada por dos horizontes de pensamiento: la teoría política y la teoría pedagógica.

Desde la teoría pedagógica, el punto de partida fue Paulo Freire y una de las herederas del pensamiento pedagógico latinoamericano, Adriana Puiggrós. A partir de la lectura de estas perspectivas, surgió la noción de "imbricación" de lo político y lo pedagógico como dos registros que se articulan mutuamente en una relación inseparable, sin que haya subordinación de un registro al otro.

A lo largo de su obra, Freire reconoce la imposibilidad de la neutralidad de lo político en lo educativo e incorpora la noción de pueblo para referirse a los campesinos, a los afrodescendientes, a los estudiantes, a los padres de familia, con lo que trasciende la noción de clase social y raza, e introduce, en Pedagogía de la esperanza (2009), el concepto de sujetos (mujeres e inmigrantes). En otras palabras, la presencia de lo político como un elemento constitutivo de los procesos educativos de los sectores populares posibilita analizar las articulaciones que se producen entre los proyectos educativos y los procesos sociales más amplios, que se vinculan con las luchas y las demandas de las organizaciones y movimientos sociales.

La mirada analítica de lo político y lo pedagógico posibilita el estudio de las relaciones de poder en los procesos de formación y constitución de sujetos y en las alternativas pedagógicas que surgen en los márgenes de la política oficial o al interior de los programas propiamente escolares y en la escuela misma.

\section{Las marcas de Freire a la educación popular para la sistematización de experiencias}

Los aportes de Paulo Freire a la educación popular y a su refundación dieron origen a formas alternas de investigación en las ciencias sociales y humanas, que hoy día son reconocidas en el campo de la investigación educativa, entre ellas la investigación participativa, la investigación-acción y la sistematización de experiencias a partir de la voz de los sujetos y con los sujetos.

En este trabajo nos referimos a la sistematización de experiencias como una práctica que ha sido recreada en proyectos comunitarios como una forma de recuperar los saberes y el conocimiento popular. En la perspectiva de la educación popular, no hay sistematización que no construya un nosotros que se conoce y que conoce, lo que no es posible si ese nosotros no está presente, como señala Ghiso (2004).

La sistematización de experiencias como una forma de investigación aporta, desde los cotidiano y lo particular, elementos esenciales al conocimiento social. Barnechea (1990) la identifica como otra vía diferente y creativa para investigar la realidad social y para aportar al conocimiento científico. Es decir, la sistematización rescata experiencias cotidianas donde se construye la sociedad y, en ese rescate, se erigen nuevos paradigmas; la sistematización se puede convertir, entonces, en un valioso aporte para esa construcción, porque permite entender las prácticas en que se ha participado para elaborar nuevos conocimientos que sirvan no solo para comprenderlas 
y mejorarlas, sino también para convertir ese conocimiento en una herramienta útil de transformación de la realidad.

De acuerdo con Mejía (2008), esta peculiar forma de investigación es una creación eminentemente latinoamericana. Lo que se entiende y practica como sistematización, en la actualidad, tiene diversas aportaciones, entre ellas el pensamiento y la huella de Paulo Freire en el marco de la educación popular. En este contexto histórico, surge un nuevo lugar de enunciación de lo teórico, en el cual los trabajos sociales, educativos e investigativos son confrontados y llevados a propuestas alternativas más acordes con las necesidades latinoamericanas.

Jara (2006) hace hincapié en que el término sistematización acompaña la visión de la educación popular. Esta última tiene sus orígenes en Brasil, con el movimiento de las comunidades de base y los Centros Populares de Cultura, en los que Paulo Freire instaló una forma de establecer las relaciones del ser humano, la sociedad, la cultura y la educación, con el concepto de concientización como condición principal y la llamada pedagogía liberadora.

De la misma manera, la educación popular materializa su sentido y planteamientos sobre el saber y el conocimiento, al abordar como una realidad la producción de conocimientos desde los mismos sujetos con quienes trabaja. Son numerosas las experiencias de sistematización y producción de teoría por parte de los sujetos populares, como fruto del diálogo entre su saber, el saber erudito y la metateoría. Para la educación popular el problema central son los procesos de producción del conocimiento a nivel local y de recreación de la vida cultural. Por eso, el educador popular no se relaciona con el conocimiento para transmitirlo, sino que se interesa por la creación de nuevos conocimientos (Mejía, 2001).

A su vez, en el campo académico, el investigador se posiciona política y pedagógicamente para incidir en la política pública, basado en la configuración de espacios socioeducativos de investigación arraigados a la realidad social y en la problemática a la que se enfrentan los grupos y organizaciones de la educación popular y de adultos, mediante el involucramiento directo de investigadores/as con la experiencia cotidiana de los sujetos, las organizaciones y los movimientos sociales.

\section{La sistematización como recuperación de la experiencia en la práctica}

La sistematización es, por lo tanto, aquello que se incluye en esa corriente ancha que busca comprender y tratar con lo cualitativo de la realidad. Ante esto, partimos de la idea inicial de que todo proceso de sistematización tiene como antecedente la práctica y la experiencia generada por un grupo, comunidad o movimiento social. Ghiso (1998) dice que esta práctica se puede recuperar, recontextualizar, textualizar, analizar y reinformar a partir de un conocimiento previo recuperado de la experiencia de la vida cotidiana.

Los conocimientos y los saberes populares, entonces, dan sentido y explican las prácticas sociales que se producen más allá de la escuela. En esa medida, la sistematización es un proceso que se realiza reflexionando sobre la práctica y buscando extraer de ella los conocimientos en que se ha sustentado, así como aquellos producidos durante la acción. Es decir, la sistematización busca sacar a la luz la teoría que está en la práctica (Barchenea, Gonzáles y Morgan Luz, 1998).

En este sentido, la sistematización — como actividad de producción de conocimientos desde la práctica - aspira a confrontar y a modificar el conocimiento teórico, gracias a una contribución que lo convierta en una herramienta útil para entender y transformar 
nuestra realidad. La sistematización produce una reconceptualización, mediante la cual las concepciones teóricas vigentes son redefinidas desde la práctica y desde los nuevos conocimientos que se elaboran al reflexionar sobre la acción. Estos nuevos conocimientos son luego difundidos y, a su vez, confrontados con otras experiencias en un proceso en espiral, flexible y dinámico, donde lo aprendido es siempre una base para nuevos conocimientos. Así, la sistematización y el nuevo saber producido irán armando un cuerpo de conocimientos, producto de la práctica, que estará en condiciones de confrontar con la elaboración teórica actualmente existente (Jara, 2003).

\section{La sistematización como producción de conocimiento}

La sistematización de experiencias parte del supuesto de que la intervención intencionada o no, en sí misma, genera un tipo de conocimiento y saberes sociales en quien la desarrolla. A este primer nivel de conocimiento se lo denomina "experiencia", y puede entenderse como equivalente al sentido común. La sistematización sería el proceso que permite transformar la "experiencia" en conocimiento ordenado, fundamentado y transmisible a otros (Francke y Morgan de la Luz, 1995).

Del mismo modo, Ghiso (1998) plantea que la sistematización es un proceso de construcción de conocimiento sobre una práctica social; este proceso no es neutro, ya aparece embuido de la construcción cultural que envuelve a la experiencia; sin embargo, conlleva la construcción de conocimiento, que además es de tipo sociocultural. Según Ghiso (1998), la propuesta de sistematización procura comprender y cualificar el quehacer social.

Podemos observar, entonces, que la sistematización es, a todas luces, un proceso de generación de conocimiento. Plantean Barchenea, Gonzáles y Morgan de la Luz (1998) que la sistematización es un proceso permanente de creación de conocimientos, siempre hecho a partir de la experiencia de intervención de una realidad social, que puede ser transformada y que es transformadora. Si la sistematización realiza una interpretación crítica del proceso de la experiencia vivida y extrae aprendizajes, quiere decir que genera un nuevo conocimiento. La cuestión verdaderamente radica en garantizar que se produzca un nuevo conocimiento a partir de los existentes.

El conocimiento siempre supone un proceso activo en el que se relaciona el saber existente con nuevas informaciones, para producir uno nuevo. La producción de conocimientos realmente nuevos supone desarrollar nuestra capacidad creativa de pensar y no solo de repetir lo que nos dicen. La sistematización de experiencias, en la medida en que tiene por objeto de conocimiento la propia experiencia, es un aspecto sustancial para la fundación de nuevos conocimientos (Jara, 2001).

\section{La sistematización como una forma de empoderar a los sujetos sociales}

Mejía (2008) considera que uno de los esfuerzos de la sistematización es no volver a pensar los espacios de la realidad social como separados o jerarquizados, sino como una forma de investigación que intenta aportar, en la ruptura, una nueva unidad que dé voz a la realidad social y contextual y, por lo tanto, nuevos elementos de movilidad social al grupo.

En efecto, en la educación popular, la sistematización es un proceso de praxis-reflexiva, a la vez que el sentido y la fuerza de su fundamentación. Por ello, los sujetos son historia, cultura, prácticas sociales; sus experiencias y vivencias son un saber que puede confluir en la educación popular. Mejía (2001) señala que en la educación popular se reconoce a los sujetos populares como dialogantes, estos confluyen al acto educativo, además de interactuar con otros, hijos de culturas, prácticas sociales y saberes diferentes, y ahí se 
construyen, entonces, nuevos saberes, conocimientos y prácticas sociales, nuevos horizontes y nuevas opciones. La sistematización se constituye, pues, en un factor de formación de nosotros/as mismos/as, de índole integral y que nos ayuda a constituirnos como sujetos críticos y creadores, desarrollando nuestras capacidades para comprender, proponer y actuar en todos los campos de la vida económica, social, política y cultural (Jara, 1998).

\section{La sistematización como investigación social}

Jara (2001) plantea que debemos resolver dialécticamente la relación entre el saber empírico y el saber científico. Señala que tenemos un paradigma de ciencia que no valora el conocimiento empírico, el saber local y solo pone énfasis en el conocimiento acumulado, en el saber constituido académicamente. Para este autor, la sistematización de experiencias es una gran posibilidad para que se expresen, desarrollen y divulguen los conocimientos y saberes locales, que tienen mucho que aportar al pensamiento científico para enriquecerlo. Esto permitirá la creación de conocimientos adecuados a la realidad que vivimos, a su mejor comprensión, y a su mejor y más cercana interpretación desde el conocimiento científico. Se deben, entonces, tomar en cuenta los conocimientos, los raciocinios, pero también las creencias, mitos, valores, emociones, todas las expresiones de nuestra subjetividad con las que impulsamos nuestras prácticas. Muchas veces se pretende obviar esto, como si fuéramos puramente razón aplicada y no seres humanos que sentimos. Esta dimensión vital e integral es decisiva para generar capacidad transformadora (Jara, 2001). Se plantea, de esta manera, la unión de estas formas cotidianas con aquella que utiliza la ciencia, pues si esta última quiere hablar de realidades sociales, no se puede desprender de la subjetividad y de la experiencia.

Aquí, señala Jara (2001), entramos en un punto clave de carácter epistemológico: la relación entre objetividad y subjetividad. El paradigma positivista pretende que no valoremos las dimensiones subjetivas, lo cual lleva a una pretendida neutralidad ante lo que sucede o ante lo que vivimos, pero esto es imposible. Lo que sí debemos buscar es objetivar lo vivido y objetivar nuestra práctica, explicitando y mirando críticamente lo que hacemos, pero no intentando ser neutrales ante ello. De ahí la riqueza de la sistematización hecha por nuestra propia práctica; esta tiene todos los elementos que se comprometen a rescatar ese conocimiento cotidiano y subjetivo, mediante un proceso riguroso y claro, como lo es la objetivación de lo vivido para poder transformarlo críticamente y mejorarlo en el futuro.

La sistematización crea nuevas relaciones entre lo particular y lo universal: lo universal no reduce lo particular a un caso; lo particular resplandece y se desenvuelve en toda su plenitud, lo que crea nuevas maneras de construir conocimiento. En este marco, la sistematización implica la revelación de lo que está oculto y de lo que está a la vista, explicitación del saber que no sabemos que sabemos, aunque sí está presente en nosotros. Cuando hablamos de explicitar o revelar somos conscientes de la fragilidad de las palabras y de que estas pueden traicionarnos; eso que vamos a revelar no está detrás, ni debajo, ni más allá; está a la vista y oculto al mismo tiempo (Messina, 2005).

\section{La sistematización como la experiencia}

La experiencia se extrae de todos los ámbitos de la vida cotidiana; basados en ella, es posible interpretar, ordenar y generar nuevas formas de entender y conocer un objeto social. Como ejemplo, citemos el caso del saber pedagógico, al cual Messina (2008) señala como el campo de la construcción que, desde la experiencia, surge y forma parte de un mundo donde es central mirar la experiencia y reflexionar sobre ella para su transformación; donde se habla de reflexión desde la práctica, de maestros reflexivos, profesionales reflexivos, profesionalidad, profesionalismo colectivo, investigación reflexiva, enseñanza reflexiva, maestros escritores, escritura como reflexión, 
comunidades de aprendizaje, sistematización educativa. De este modo, se establece un vínculo estrecho entre reflexión, constitución de la profesión docente, formación e investigación; todas estas categorías giran en torno de la construcción del saber pedagógico a partir de la experiencia.

Concebir de esta manera el saber pedagógico pone al sujeto en un lugar prominente; la pedagogía, entonces, deja de ser algo que está fuera de los educadores, un saber objetivado, un cuerpo teórico construido históricamente por personajes notables; la pedagogía empieza a ser un patrimonio de todos los educadores, de todos aquellos anónimos e innumerables, legitimados por su trabajo. Hablar de saber pedagógico desde la experiencia no es negar la concepción de una pedagogía constituida como disciplina, con reglas de juego, con autonomía teórica y metodológica, inscrita en un mundo social, vinculada con la Modernidad, sino abrir la posibilidad de que los educadores dialoguen con ella desde sus propios saberes. Al mismo tiempo, un saber pedagógico que se construye desde la experiencia permite pensar la relación entre saber y poder, entender la producción de saber como un proceso enmarcado en líneas de autoridad, organizado con una lógica de centro, así como desplazarse de una visión tradicional del currículo como programa, como oferta, hacia un sistema de vínculos, sujetos, diálogos, conversaciones, significados y significantes que forman una trama compleja y densa (Messina, 2008).

La sistematización ordena las experiencias, pero pasar de ordenar una experiencia a decantar sus enseñanzas requiere una reconstrucción descriptiva, aunque hay que ir más allá: es preciso someterla a reflexión y análisis. La sistematización adquiere el carácter de actividad teórico-práctica, puesto que la interpretación y la comprensión de la experiencia son imposibles sin referirla, de un lado, a los supuestos teóricos a partir de los cuales se proyectó la intervención y, del otro, al conocimiento empírico y teóricoconceptual existente. El proceso solo culmina cuando se formalizan los aprendizajes obtenidos y se comparten y contrastan con los producidos a partir de experiencias similares (Francke y Morgan de la Luz, 1995).

Finalmente, a partir de los diversos sentidos que adquiere la sistematización, lo fundamental para este escrito es la herencia político-pedagógica de Paulo Freire. Los autores citados se formaron y fueron creadores de la corriente de la educación popular, son educadores populares e intelectuales comprometidos con las luchas sociales en nuestra América Latina.

Además, configuraron la sistematización como una manera de realizar investigación social con y desde los sectores populares. De acuerdo con Jara (2003), lo más característico y propio de la reflexión sistematizadora sería que busca permanecer, así como meterse por dentro de esos procesos sociales vivos y complejos, circula entre sus elementos, palpa las relaciones entre otros, recorre sus diferentes etapas, localiza sus contradicciones, marchas y contramarchas hasta entender estos procesos desde su propia lógica, y a partir de allí extrae enseñanzas que puedan aportar al enriquecimiento tanto de la práctica como de la teoría.

\section{La sistematización de experiencias y su articulación con el posicionamiento político-pedagógico}

\section{Antecedentes}

A lo largo de mi trayectoria profesional en el campo de la educación popular y de adultos, en las organizaciones y los movimientos sociales ha permeado la perspectiva de la sistematización de experiencias a manera de investigación social, que parte de la 
voz de los sujetos y de su propia narrativa para ir configurando de manera colectiva o individual, ¿cómo el sujeto resignifica la propia historia a partir de su inserción en las organizaciones y en la lucha social?, ¿cómo se potencia como sujeto en lo individual y en lo colectivo en esa lucha?, ¿qué perspectivas de futuro va construyendo para transformar el mundo y la realidad?

Sin duda, la participación en el Proyecto de Alternativas Pedagógicas y Prospectiva Educativa en América Latina (APPEAL) representó un momento clave para problematizar el análisis y la sistematización de las alternativas pedagógicas: alternativas a qué, en qué contextos y con cuáles condiciones de posibilidad. Esta mirada se vio enriquecida con el horizonte de pensamiento de Ernesto Laclau, con la resignificación y la deconstrucción de la noción de hegemonía y la operación política a través de procesos de articulación y antagonismo para entender cómo los significantes flotantes operan políticamente.

En este contexto, la lectura de Paulo Freire en la Facultad de Filosofía y Letras de la Universidad Nacional Autónoma de México ofrecía una mirada pedagógica desde un horizonte político. Freire, además de ser latinoamericanista, ofrecía herramientas teóricas y metodológicas del hacer y quehacer de la educación a partir de la palabra generadora para transformar el mundo y la realidad. De esta manera, lo político y lo pedagógico se fueron configurando como un horizonte de inteligibilidad (Freire, Puiggrós y Laclau).

Mi formación en el ámbito de la investigación educativa en el Departamento de Investigaciones Educativas (DIE), a través de mi tesis doctoral, me dio la oportunidad de operar este horizonte de inteligibilidad de la imbricación dentro de lo político y lo pedagógico y la construcción de un concepto intermedio, el archipiélago educativo, con el propósito de dar cuenta de los procesos educativos que se dan más allá de la escuela, de los saberes y aprendizajes sociales que se construyen en el mundo de vida de los sujetos para la resolución de sus problemas y necesidades inmediatas: el trabajo, la familia, la asamblea comunitaria, entre otros.

Para la construcción de esta noción fue de mucha utilidad el texto Mil mesetas, de Deleuze y Guattari (1994), porque me permitió dar cuenta de los múltiples espacios de formación y constitución de sujetos. Se trata de espacios que no son fijos, estables, ni independientes, en los que se desplazan sentidos políticos y pedagógicos: participación, solidaridad, comunalidad, entre otros.

Con este andamiaje documenté dos experiencias educativas que surgen en los márgenes de la política oficial, lo que me permitió problematizar la educación más allá de la escuela y centrar mi atención en los procesos de educación de adultos que emergen desde la educación popular. Por ende, interesaba dar cuenta de la constitución de sujetos, la construcción de agencia y la transformación social del entorno más inmediato: la familia y las comunidades.

Con este capital, y desde el ámbito de la investigación educativa, promoví una línea de investigación en la Universidad Iberoamericana acerca de Educación de Adultos y Organizaciones Civiles, y realicé investigación social desde la sistematización de experiencias a través de entrevistas, grupos focales y análisis de documentos. Entre los trabajos realizados, cabe mencionar los siguientes proyectos: Sistematización de experiencias de las Plazas Comunitarias, Unión y fuerza 19 de septiembre, Organización Unión Popular Revolucionaria Emiliano Zapata y Cooperativa de Mujeres para Hoy.

En los últimos años destacan dos trabajos referidos a la sistematización de la práctica: educación, interculturalidad y organización popular Tseltal, a más de cincuenta años 
de la presencia de los jesuitas en Chiapas, México, en particular la sistematización de la Organización Social Yomlej, construcción y experiencia desde la base y, la sistematización de los saberes políticos y pedagógicos desplegados por los estudiantes de la Ibero en el movimiento estudiantil Más de 131 y \#Yosoy132. Ambos proyectos fueron sistematizados y documentados por los propios sujetos involucrados a través de la remembranza de la memoria histórica por medio de entrevistas colectivas en grupos focales.

\section{La experiencia más reciente}

La sistematización y recontrucción de la experiencia de los saberes y los aprendizajes sociales desplegados por los estudiantes que participaron en el movimiento estudiantil Más de 131 y \#Yosoy132, en la Univeridad Iberoamericana de la Ciudad de México fue la experiencia más reciente. Para la sistematización de la misma se hizo un llamdo a los estudiantes que participaron en el movimiento para re-vivir las acciones, las emociones $y$ sentires en torno al movimiento.

La respuesta al llamado por parte de los estudiantes se dio de manera libre y abierta al diálogo, a la manera de grupo focal, y se partió de tres preguntas para iniciar las charlas referidas a las acciones colectivas de los estudiantes universitarios: qué pasó aquel 11 de mayo de 2012, en la Ibero, qué recuerdos vienen a su memoria y qué emociones se generaron. Al frente del grupo focal participábamos tres personas: una estudiante de la maestría en Investigación y Desarrollo de la Educación, un estudiante de doctorado del Programa Doctoral Interinstitucional en Educación del Sistema Universitario Jesuita, que investigaba al respecto, y yo como experta en sistematización, entrevistas colectivas y disparadora de ideas para el diálogo.

Poco a poco los estudiantes empezaron a activar su memoria de aquel 11 de mayo de 2012; siguen señalando, aún con asombro y emoción, que no esperaban la respuesta de los estudiantes de la Ibero, pero tampoco la de las autoridades universitarias, la de otras universidades privadas y públicas y la forma en que la ciudadanía modificó la mirada sobre la Universidad Iberoamercana y sus estudiantes; todavía queda el recuerdo del día en que fueron invitados a la UNAM, la universidad pública de mayor importancia en la vida social del país, y fueron recibidos con un enorme aplauso y con Goyas para los estudiantes de la Ibero, dejando atrás los estigmas de "niños fresas, niños bien, niños de papi”, entre otros.

Emocionaba ver la solidaridad que se creó entre estudiantes de universidades privadas y públicas, todos unidos en el descontento general, en el deseo de ver un cambio en la idiosincrasia de la hegemonía política del momento y evidenciar la manipulación de los medios de comunicación.

Con la narrativa de los estudiantes se elaboraron ejes temáticos a manera de estampas del Movimiento estudiantil Más de 131 y \#Yosoy132, para que ellos, en lo individual y en lo colectivo, continuaran con el relato de sus experiencias en el movimiento estudiantil, sus saberes y sus sentires. A partir de ese momento, con los ejes temáticos que emergieron del diálogo inicial, se acompañó a los estudiantes en la escritura de sus relatos haciendo puntualizaciones y señalizaciones para profundizar hasta contar con un texto para su difusión en el marco de una investigacion acerca de la irrupción estudiantil en la Ibero, una universidad privada, jesuita, con inspiración en la filosofía ignaciana acerca de la justicia, el compromiso y el cambio social.

Con esta bandera en el corazón, el movimiento rompió las paredes de la desinformación y la apatía social; expresó sus anhelos y exigencias mediante marchas, gritos, porras, asambleas, desveladas, tinta en cartulina, puño alzado, fotografías, videos, 
facebookazos y twittazos que levantaron a la juventud dormida y unieron a los estudiantes fragmentados.

Llama la atención la contrucción de la narrativa por parte de los estudiantes y su creatividad para recibir ese 11 de mayo de 2012, al candidato a la presidencia de la República Enrique Peña Nieto, del Partido Revolucionario Institucional (PRI):

Aquel 11 de mayo de 2012, en el auditorio José Sánchez Villaseñor de la Ibero, se percibía un esfuerzo del equipo de Peña por controlar cada detalle, hasta los mensajes comunicados en fotografías... pero fue imposible contener al centenar de estudiantes que mostraban su descontento con el candidato y lo que este representaba: a través de pancartas, máscaras de Salinas de Gortari, rostros rojos “bañados en sangre”, los posters de "\#contigohastalospinos" no pudieron esconder los reclamos escritos: "Atenco no se olvida", “No más feminicidios" o "EPN, la lbero no te quiere”, de la mayoría de los estudiantes.

En síntesis, la sistematización de experiencias a partir de la voz de los integrantes de un movimiento estudiantil, una cooperativa o una experiencia docente permiten dar cuenta de las acciones colectivas, los saberes y los sentires que se construyen en torno a las alternativas pedagógicas de nuestra América Latina y de la lucha político-pedagógica de Paulo Freire: aprender a leer la realidad, recrearla y transformarla.

\section{A manera de cierre}

» Paulo Freire es, sin duda, uno de los pensadores más importantes del siglo XX, con un gran impacto en diversos contextos de la aldea global. Es punto de referencia en las pedagogías críticas y emancipatorias. La lectura del mundo y de la realidad para su transformación se han convertido en dispositivos clave del hacer y el quehacer político-pedagógico en el espacio escolar y fuera de la escuela. El legado para el pensamiento latinoamericano en diversas áreas del conocimieno ha sido fundamental y ha acompañado a diversos movimientos sociales.

» En América Latina, los registros de lo político y lo pedagógico inscritos en la narrativa educativa han representado el posicionamiento ético, ideológico y político de que todo acto educativo es político y viceversa. En el caso de quien suscribe, representa una manera de alargar la mirada para analizar los procesos sociales que transcurren en múltiples espacios sociales, lo cual permitió construir una categoría intermedia, el archipiélago educativo.

» La sistematización de experiencias surge, en América Latina, gracias a la herencia del pensamiento pedagógico freireano. La generación formada en el ámbito de la educación popular, la misma que acompañó las luchas de los movimientos sociales, vio en la sistematización de experiencias una manera de construir el conocimiento y los saberes populares que se configuran en la misma lucha. Sin duda, la sistematización de experiencias representa una corriente de investigación social que ha sido recreada desde diferentes lugares, grupos de trabajo, universidades y educadores populares con la inspiración y al cobijo de la obra de Paulo Freire.

» La reconstrucción y la sistematización de experiencias a partir de la voz de los sujetos representan una opción político-pedagógica para el quehacer de la investigación. En lo personal, representan un posicionamiento y un horizonte de inteligibilidad porque alargan la mirada a los procesos educativos que transcurren en múltiples espacios de formación y constitución de sujetos. 


\section{Bibliografía}

» Barnechea, M. (1990). La importancia de la Sistematización en la Construcción de una Nueva Sociedad. Universidad de Arte y Ciencias Sociales ARCIS. Disponible en: http://www.mag-politicasociales.cl/documentos/m_barnechea1.pdf

" Barchenea, M.; Gonzáles, E. y Morgan Luz, M. (junio de 1998). La Producción de Conocimientos en Sistematización. Disponible en: http://www.alboan.org/ archivos/339.pdf

»Bello-Urrego, A. (2013). Sexo/género, violencias y derechos humanos: perspectivas conceptuales para el abordaje de la violencia basada en género contra las mujeres desde el sector salud. Revista Colombiana de Psiquiatría, 42(1), 108-119.

》Deleuze, G. y Guattari, F. (1994). Mil mesetas: capitalismo y esquizofrenia. Pretextos.

»De Sousa, B. (2009). Una epistemología del sur. La reivindicación del conocimiento y la emancipación social. Buenos Aires, Siglo XXI-CLACSO.

»Dubar, C. (2002). La crisis de las identidades. La interpretación de una mutuación. Barcelona, Bellatera.

» Francke, M. y Morgan de la Luz, M. (octubre de 1995). La Sistematización: apuesta por la generación de conocimientos a partir de las experiencias de promoción. Lima, Escuela para el desarrollo. Disponible en: http://www.alboan.org/archivos/343.pdf

》 Freire, P. (1997). Pedagogía de la esperanza. México, Siglo XXI.

»Freire, P. (2006). Cartas a quien pretende enseñar. México, Siglo XXI.

»Freire, P. (2019). Pedagogía del oprimido. México, Siglo XXI.

» Ghiso, A. (1998). De la práctica singular al diálogo con lo plural. Medellín, FUNLAM.

» Ghiso, A.; Mejía, M.; Mariño, G.; Torres, A. y Cendales, L. (2004). Sistematización de Experiencias. Propuestas y Debates. Bogotá, Dimensión Educativa.

» Jara, O. (1998). El aporte de la Sistematización a la Renovación Teórico Práctica de los Movimientos Sociales. Ponencia para el Seminario Latinoamericano: Sistematización de Prácticas de Animación Sociocultural y Participación Ciudadana. Medellín. Agosto. Disponible en: Alforja, programa coordinado de educación popular: www.alforja. or.cr/sistem/Jara.doc

"Jara, O. (2001). Dilemas y desafíos de la sistematización de experiencias. Costa Rica, CEP Centro de Estudios y Publicaciones Alforja.

» Jara, O. (2002). El Desafío Político de Aprender de Nuestras Prácticas. Diálogos: Educación y formación de personas adultas, 2(42-43), 59-64. Disponible en: http:// www.alforja.or.cr/sistem/aprenderdepracticas.pdf

» Jara, O. (2003). Para sistematizar experiencias. INNOVANDO (20). Chile, Equipo de Innovación Educativa. Recuperado de Dirección General de Educación Superior y Técnico Profesional de Perú. Disponible en: http://destp.minedu.gob.pe/ Secundaria/nwdes/pdfs/revistaie2o.pdf

"Jara, O. (2006). La sistematización de experiencias y las corrientes innovadoras del pensamiento latinoamericano. Una aproximación histórica. Diálogo de Saberes (3), 118-129. Disponible en: http://www.planificacionparticipativa.upv.es/wordpress/ wp-content/uploads/2011/o6/La-sistematizacion-de-experiencias-y-las-corrientesinnovadoras-del-pensamiento-latinoamericano-una-aproximacion-historica.pdf 
" Magaña, H. (2014). Jóvenes y Derechos. Alegatos-Revista Jurídica de la Universidad Autónoma Metropolitana, (87), 327-350.

» Mejía, R. M. (2001). Pedagogía en la Educación Popular. Congreso Pedagógico Nacional de Fe y Alegría. Bogotá, Fe y Alegría.

" Mejía, R. M. (2008). Atravesando el Espejo de Nuestras Prácticas. Biblioteca Virtual sobre Sistematización de Experiencias. Programa Latinoamericano de Sistematización de Experiencias del CEAAL. Disponible en: http://www.cepalforja. org/sistem/bvirtual/?p=715

» Mejía, R. M. (2008). La Sistematización como proceso investigativo. O la búsqueda de la episteme de las prácticas. Ampliación del editorial. Revista Internacional Magisterio, (33). Junio-julio 2007. Disponible en: http://www.cepalforja.org/sistem/sistem_old/ sistematizacion_como_proceso_investigativo.pdf

" Messina, G. (2005). La Sistematización: acerca de su especificidad. Revista Interamericana de Educación de Adultos, 27(2): 163-171.

"Messina, G. (2008). Construyendo saber pedagógico desde la experiencia. Biblioteca Virtual sobre Sistematización de Experiencias. Programa Latinoamericano de Sistematización de Experiencias del CEAAL. Disponible en: http://www.cepalforja. org/sistem/bvirtual/?p=719

»Pérez-León, J. P. (2008). El individuo como sujeto de derecho internacional. Análisis de la dimensión activa de la subjetividad jurídica internacional del individuo. Anuario mexicano de derecho internacional, (8): 599-642. Disponible en: http://www.scielo. org.mx/scielo.php?script=sci_arttext\&pid=S1870-46542008000100018\&lng=es\&tln $\mathrm{g}=\mathrm{es}$

» Ruiz, D. (2001). Sistematización de Prácticas. Sistematización. Experiencia de convivencia. Liceo Nacional Marco Fidel Suárez. Disponible en: https://www.google. $\mathrm{com} / \mathrm{search}$ ?q=Ruiz+Botero\%2C+D.+(2001). + Sistematizaci\%C $3 \%$ B $3 n+$ de $+\operatorname{Pr} \% \mathrm{C}_{3}$ $\%$ A1cticas.\&oq=Ruiz+Botero\% 2 C + D. $+(2001) .+$ Sistematizaci\%C $3 \%$ B $3 n+d e+\operatorname{Pr} \% C$ $3 \%$ A1cticas.\&aqs=chrome..69i57.463joj4\&sourceid $=$ chrome \&ie $=U T F-8$

»Ruiz, D. (2005) Imbricación de lo político y lo pedagógico en los procesos de educación de adultos. México, Centro de Cooperación Regional para la Educación de Adultos en América Latina y el Caribe.

» Ruiz, M. y Araujo, C. (2018). (coords.) Insurrección estudiantil y acción ciudadana: Más de 131 y \#Yo soy 132. México, Universidad Iberoamericana/ Sistema Universitario Jesuita.

\section{María Mercedes Ruiz Muñoz}

Doctora en Ciencias con especialidad en Investigación Educativa. Pedagoga con especialidad en sociología de la educación. Académica e investigadora de la Universidad Iberoamericana (México). Integrante del Sistema Nacional de Investigadores. Investigación en Derecho a la educación, política y justicia escolar, Educación de adultos y organizaciones civiles. mercedes.ruiz@ibero.mx 